\title{
Effect of drillpipe rotation on cuttings transport using computational fluid dynamics (CFD) in complex structure wells
}

\author{
Xiaofeng Sun · Kelin Wang · Tie Yan • \\ Shuai Shao $\cdot$ Jianjun Jiao
}

Received: 11 May 2013/Accepted: 5 May 2014/Published online: 29 May 2014

(C) The Author(s) 2014. This article is published with open access at Springerlink.com

\begin{abstract}
Effective cuttings transport is one of the most major concerns in designing hydraulic parameters during drilling complex structure wells. It is important and essential to study critical parameters how to influence cuttings bed development, especially the drillpipe rotation effects on cuttings transport behavior. In this study, cuttings transport simulations were carried out for inclinations from $45^{\circ}$ to $90^{\circ}$, for pipe rotation speeds from 80 to $240 \mathrm{rpm}$, and for flow rates from 30 to $50 \mathrm{~L} / \mathrm{s}$. Annular cuttings volume, pressure drop, and cuttings distribution are recorded simultaneously within the simulation proceeding. The CFD simulations show that pipe rotation makes cuttings presented in asymmetric distribution along the wellbore and significantly improves the drag effects on drilling fluid in the tangential direction. At a low or medium flow rate, pipe rotation has a significant impact on annular cuttings volume and pressure drop. However, there is no additional contribution of pipe rotation after reaching critical speed at high flow rates. Buckingham- $\pi$ theorem combined with least square method was applied to establish the empirical correlations for estimating cuttings concentration and annular pressure drop.
\end{abstract}

Keywords CFD - Hole cleaning - Cuttings transport . Drillpipe rotation $\cdot$ Complex structure well

X. Sun $\cdot$ K. Wang $(\bowtie) \cdot$ T. Yan $\cdot$ S. Shao

College of Petroleum Engineering, Northeast Petroleum

University, Daqing 163318, China

e-mail: wwangkelin@163.com

J. Jiao

Tianjin Branch of CNPC Offshore Engineering Co. Ltd,

Tianjin 300450, China

\section{Introduction}

Complex structure well includes horizontal well, extended reach well, and multilateral well, which has been widely used for offshore oil exploration and exploitation and improving the recovery of oil field. However, hole cleaning is often difficult to be handled during drilling inclined and horizontal sections of complex structure wells, and cuttings are easily accumulated to develop cuttings bed, especially in the inclinations around $40^{\circ}-60^{\circ}$, which may lead to various problems such as high drag and torque, pipe stuck, weight stacking, higher hydraulic requirements and excessive ECD, and cementing issues. Therefore, it is important and essential to study the key factors of cuttings bed development in annulus, especially if drillpipe is rotating. Numerous researches related to the effect of pipe rotation on hole cleaning have been carried out by experimental investigations and CFD simulations in the past several years.

In experimental investigations, Peden et al. (1990) observed that pipe rotation has a significant effect on the minimum transport velocity in medium or highly viscous fluids and contributes to cuttings transport in small annulus or positive eccentricity. Sifferman et al. (1992) pointed out that drillpipe rotation reduces annular cuttings buildup under certain conditions, and the effect is greatest at inclination near horizontal, for small cuttings and low rate of penetrations. Bassal (1996) indicated pipe rotation enhances hole cleaning more when the used mud has a higher viscosity with smaller cuttings sizes or for hole angle at $65^{\circ}$. Sanchez et al. (1999) analyzed the effects of pipe rotationflow rate, pipe rotation-mud rheology, pipe rotation-inclination, and pipe rotation-cuttings size. The results show that in lower inclinations, no critical range of rotary speeds is determined but higher meant better, and orbital motion 
Fig. 1 Physical model of inclined section

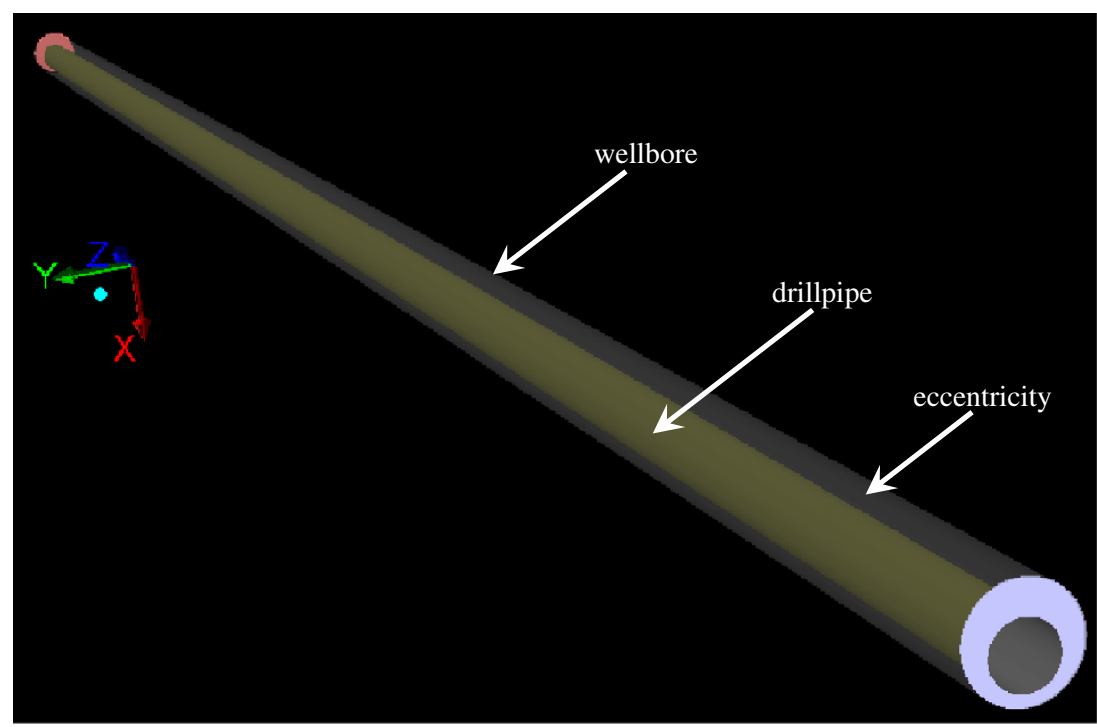

can significantly improve cuttings transport. Duan et al. (2008) performed the experiments about different cuttings sizes under pipe rotation. It was observed that small cuttings transport is mainly dominated by pipe rotation and fluid rheology, and improvement by pipe rotation in the transport efficiency of small cuttings is up to twice as large as the improvement in large cuttings transport. Ozbayoglu et al. (2008) and Sorgun (2010) indicated pipe rotation has a significant improvement on cuttings transport, especially when the pipe is making an orbital motion. But drillpipe rotation has no additional contribution to hole cleaning after a certain rotation speed. Also, the empirical correlations for $60^{\circ}-90^{\circ}$ were developed for predicting the stationary cuttings bed height and frictional pressure drop.

In CFD simulations, Bilgesu et al. $(2002,2007)$ were one of the first researchers to analyze cuttings transport parameters using CFD. The simulations show drillpipe rotation can improve the cuttings transport, and the effect is more pronounced for smaller particle size. Mishra (2007) found that hole cleaning efficiency is improved more significantly for small cuttings than for large cuttings as pipe rotation is increased, and pipe rotation at a fixed velocity can decrease pressure loss. Wang et al. (2009) indicated the solid flow becomes favorable, and the solid volume is reduced with the increase of rotary speeds. The correlation was also developed to estimate cuttings bed height. Li et al. (2010) found that pipe rotation between 80 and $120 \mathrm{rpm}$ has a significant effect on hole cleaning.

Although some achievements have been made, but the pipe rotation effects on cuttings transport are difficulty be accurately depicted due to the complexity of cuttings-fluid coupling. The purpose of this paper mainly further discussed the cuttings transport behavior with pipe rotation using CFD.

\section{Physical model of inclined section}

Complex structure well usually contains the two or three buildup sections. The $215.9 \mathrm{~mm}$ wellbore diameter and $127 \mathrm{~mm}$ outer diameter of drillpipe are adopted to simulate the actual annulus. A 12-m-long interval is simulated without considering borehole enlargement and shrinkage. The eccentricity is 0.5 , and eccentric distance is $44.45 \mathrm{~mm}$. The gravity effect, horizontal and vertical vibrations are also considered, and the diagram of the physical model is shown in Fig. 1.

\section{Gridding}

To simulate the effect of drillpipe rotation on transient cuttings transport in annulus, moving mesh is applied for the flow domain in annulus. As shown in Fig. 2, all the annulus can be divided into both outer and inner flow domain. The flow domain near drillpipe is rotating with a certain speed to simulate the pipe rotation effect, and the outer flow domain is static. In addition, inner flow domain transfers pressure, velocity, cuttings concentration, and other parameters through interface.

\section{Mathematical model of CFD simulations}

To observe the effect of different rotation speeds on the drilling fluid-cuttings two-phase flow behavior, the Euler multiphase flow in rotary coordinate system is chosen as the simulated model. Standard function is used to calculate the momentum equation for low Reynolds number zone near drillpipe surface. The momentum equation for fully turbulent zone is determined by Realizable $\kappa-\varepsilon$ model (Shih 
Fig. 2 Side view and profile of annular grid structure

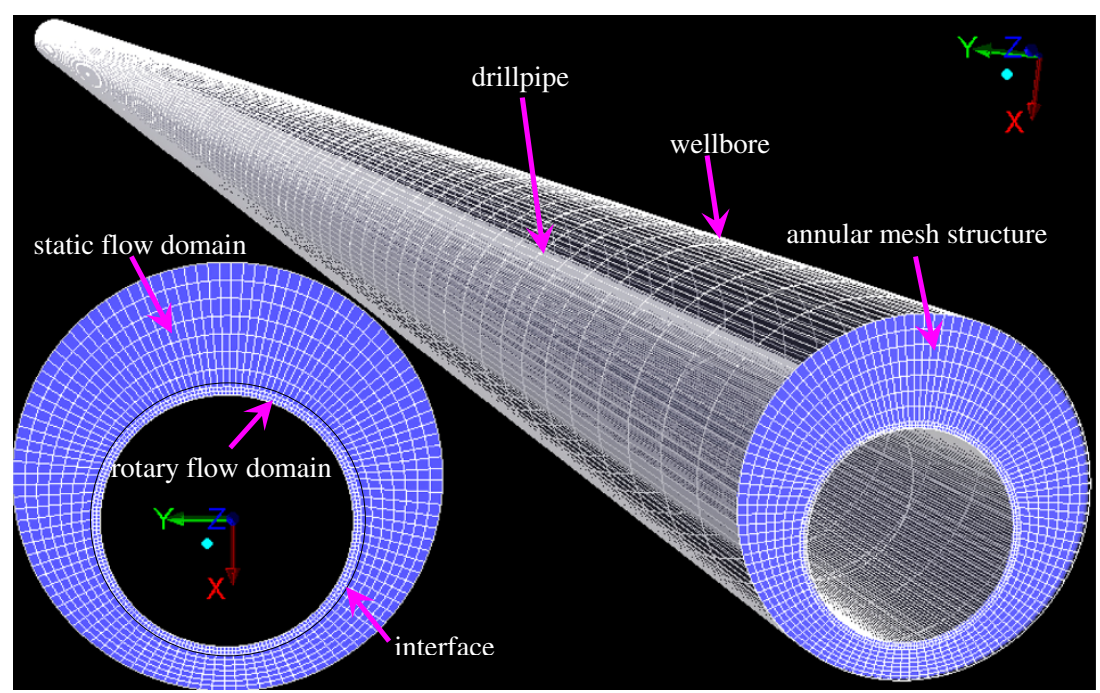

et al. 1995). Also, first order upwind scheme of finite volume method is applied to discretize the governing equations, and the value that settling cuttings volume in annulus tends to be stable is considered as convergence criterion. These governing equations can be listed as follows:

Mass equation of liquid phase:

$\frac{\partial}{\partial t}\left(\alpha_{l} \rho_{l}\right)+\nabla \cdot\left(\alpha_{l} \rho_{l} \vec{v}_{l}\right)=\Delta m_{s l}$

Mass equation of solid phase:

$\frac{\partial}{\partial t}\left(\alpha_{s} \rho_{s}\right)+\nabla \cdot\left(\alpha_{s} \rho_{s} \vec{v}_{s}\right)=\Delta m_{l s}$

Momentum equation of liquid phase:

$$
\begin{aligned}
& \frac{\partial}{\partial t}\left(\alpha_{l} \rho_{l} \vec{v}_{l}\right)+\nabla \cdot\left(\alpha_{l} \rho_{l} \vec{v}_{l} \vec{v}_{l}\right)+\rho_{l}\left(2 \vec{\omega} \times \vec{v}_{l}+\vec{\omega} \times \vec{\omega} \times \vec{r}\right) \\
& =-\alpha_{l} \nabla p+\mu \nabla^{2} \vec{v}_{l}+\alpha_{l} \rho_{l} \vec{g} \\
& \quad+K_{s l}\left(\vec{v}_{s}-\vec{v}_{l}\right)+\Delta m_{s l} \vec{v}_{s l}-\Delta m_{l s} \vec{v}_{l s}
\end{aligned}
$$

Momentum equation of solid phase:

$$
\begin{aligned}
& \frac{\partial}{\partial t}\left(\alpha_{s} \rho_{s} \vec{v}_{s}\right)+\nabla \cdot\left(\alpha_{s} \rho_{s} \vec{v}_{s} \vec{v}_{s}\right)+\rho_{s}\left(2 \vec{\omega} \times \vec{v}_{s}+\vec{\omega} \times \vec{\omega} \times \vec{r}\right) \\
& =-\alpha_{s} \nabla p+\mu \nabla^{2} \vec{v}_{s}+\alpha_{s} \rho_{s} \vec{g} \\
& \quad+K_{s l}\left(\vec{v}_{l}-\vec{v}_{s}\right)-\Delta m_{s l} \vec{v}_{s l}+\Delta m_{l s} \vec{v}_{l s}
\end{aligned}
$$

where " $s$ " and " $l$ " are the subscripts that represent solidand liquid-phase parameter. $\Delta m_{s l}$ is that the mass transfers from solid phase to fluid phase. Similarly, the position exchange of subscript indicates the mass transfers from fluid phase to solid phase. Also, $\Delta m_{s l}=-\Delta m_{l s} ; v_{l s}$ is the velocity between phases. When $\Delta m_{l s}>0, v_{l s}=v_{s l}$. When
$\Delta m_{l s}<0, v_{l s}=v_{\mathrm{s}}$ and $v_{l s}=v_{s l} ; \alpha$ is the phase volume fraction; $K_{s l}$ is the solid-liquid momentum interchange coefficients, and $K_{s l}=\alpha_{\mathrm{s}} \rho_{\mathrm{s}} f / \tau_{\mathrm{s}} \cdot \tau_{\mathrm{s}}$ is the relaxation time of particles, and $\tau_{\mathrm{s}}=\rho_{\mathrm{s}} d_{\mathrm{s}}^{2} /(18 \mu)$. Here, $K_{l s}=K_{s l}$, and $f$ which can be obtained from Syamlal-O'Brien model (Syamlal et al. 1989) is the function of drag force.

\section{Boundary conditions and initial conditions}

Boundary conditions of the transient simulation apply velocity inlet and pressure outlet, and setting different values of inlet velocity and outlet pressure can be used to simulate the different rotation speeds and well depths, respectively. The density of drilling fluid is $1,200 \mathrm{~kg} / \mathrm{m}^{3}$, and the viscosity is $30 \mathrm{mPa}$ s. Cuttings density is $2,500 \mathrm{~kg} / \mathrm{m}^{3}$, and the diameter is $8 \mathrm{~mm}$. The volume fraction of cuttings is $1 \%$. The simulations are divided into 12 groups, and the more detailed parameters are shown in Table 1.

\section{Results and discussions}

Effects of pipe rotation on velocity profile

The purpose of analyzing the velocity profile is that the effects of pipe rotation on annular pressure drop are actually caused by redistribution in annulus. The redistribution of velocity also has a significant effect on cuttings transport. As shown in Fig. 3, CFD simulations show that tangential velocity is decreases by exponential function along the tangential direction and the ratio of reduction become small with the increase of rotation speed. It is generally considered that the tangential velocity of drilling fluid is 
Table 1 The variables required by CFD simulations

\begin{tabular}{|c|c|c|c|}
\hline Groups & $\begin{array}{l}\text { Inclination } \\
\left({ }^{\circ}\right)\end{array}$ & $\begin{array}{l}\text { Flow rate } \\
(\mathrm{L} / \mathrm{s})\end{array}$ & Pipe rotation speed (rpm) \\
\hline $1-9$ & 45 & 30 & $\begin{array}{l}80,100,120,140,160,180,200 \\
\quad 220,240\end{array}$ \\
\hline $10-18$ & 45 & 40 & $\begin{array}{l}80,100,120,140,160,180,200 \\
\quad 220,240\end{array}$ \\
\hline $19-27$ & 45 & 50 & $\begin{array}{l}80,100,120,140,160,180,200 \\
220,240\end{array}$ \\
\hline $28-36$ & 60 & 30 & $\begin{array}{l}80,100,120,140,160,180,200 \\
220,240\end{array}$ \\
\hline $37-45$ & 60 & 40 & $\begin{array}{l}80,100,120,140,160,180,200 \\
220,240\end{array}$ \\
\hline $46-54$ & 60 & 50 & $\begin{array}{l}80,100,120,140,160,180,200 \\
\quad 220,240\end{array}$ \\
\hline $55-63$ & 75 & 30 & $\begin{array}{l}80,100,120,140,160,180,200 \\
220,240\end{array}$ \\
\hline $64-72$ & 75 & 40 & $\begin{array}{l}80,100,120,140,160,180,200 \\
\quad 220,240\end{array}$ \\
\hline $73-81$ & 75 & 50 & $\begin{array}{l}80,100,120,140,160,180,200 \\
220,240\end{array}$ \\
\hline $82-90$ & 90 & 30 & $\begin{array}{l}80,100,120,140,160,180,200 \\
\quad 220,240\end{array}$ \\
\hline $91-99$ & 90 & 40 & $\begin{array}{l}80,100,120,140,160,180,200 \\
\quad 220,240\end{array}$ \\
\hline $100-108$ & 90 & 50 & $\begin{array}{l}80,100,120,140,160,180,200 \\
\quad 220,240\end{array}$ \\
\hline
\end{tabular}

generated near drillpipe surface with drillpipe rotation. The cuttings are tangentially dragged by viscosity force of drilling fluid, which can improve hole cleaning. However, due to the reduction of tangential velocity of drilling fluid, the drag force acting on cuttings gradually becomes less and some cuttings can not be effectively transported into main flow field. As a result, these cuttings will settle at the bottom of annulus and eventually cuttings bed is formed. This indicates high rotation speed can provide more energy for suspending the cuttings, makes most cuttings fully suspended, and decrease cuttings bed height.

\section{Distribution of annular cuttings with pipe rotation}

The simulations on the distribution of cuttings in annulus for angle at $45^{\circ}$ and $60^{\circ}$ were conducted, and the following conclusions can be reached by comparing Fig. 4 with 5:

- Cuttings present asymmetrical distribution on either side of drillpipe, and cuttings bed is easy to be formed when low mud flow rate is used.

- As drillpipe rotation speed increases, cuttings concentration in annulus decreases for any pipe rotation speed, but the level of enhancement is more pronounced with the decrease at flow rate.

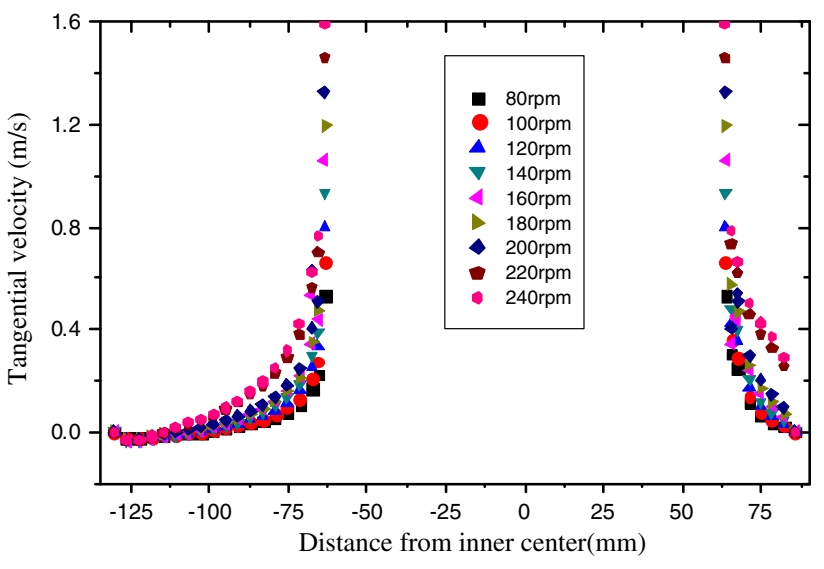

Fig. 3 Tangential velocity profile in an eccentric annulus with pipe rotation

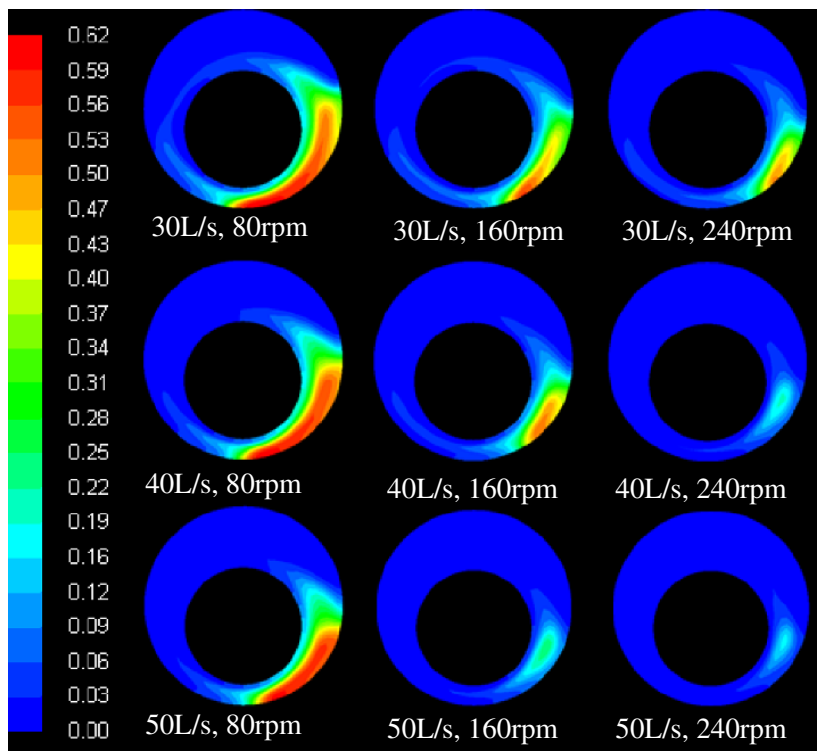

Fig. 4 Annular cuttings concentration contour at the angle of $45^{\circ}$

- Lower cuttings concentration near drillpipe are presented, which indicates that drilling fluid can obtain high tangential velocity under the drillpipe rotation, and cuttings are suspended by high drag force and transported by axial fluid flow.

- In the low flow rate, cuttings are more transported at the angle of $60^{\circ}$ than $45^{\circ}$, but inclination shows only a minor effect on cuttings concentration in high flow rate and rotation speed.

- Pure drilling fluid region, dispersed layer, and stationary cuttings bed are emerged in low rotary speed or flow rate. As the flow rate is increased, the intensity of turbulent eddies grows in strength and eventually the particles of the cuttings bed are totally lift into the dispersed layer and there only exists pure drilling fluid layer and dispersed layer. Finally, with further 


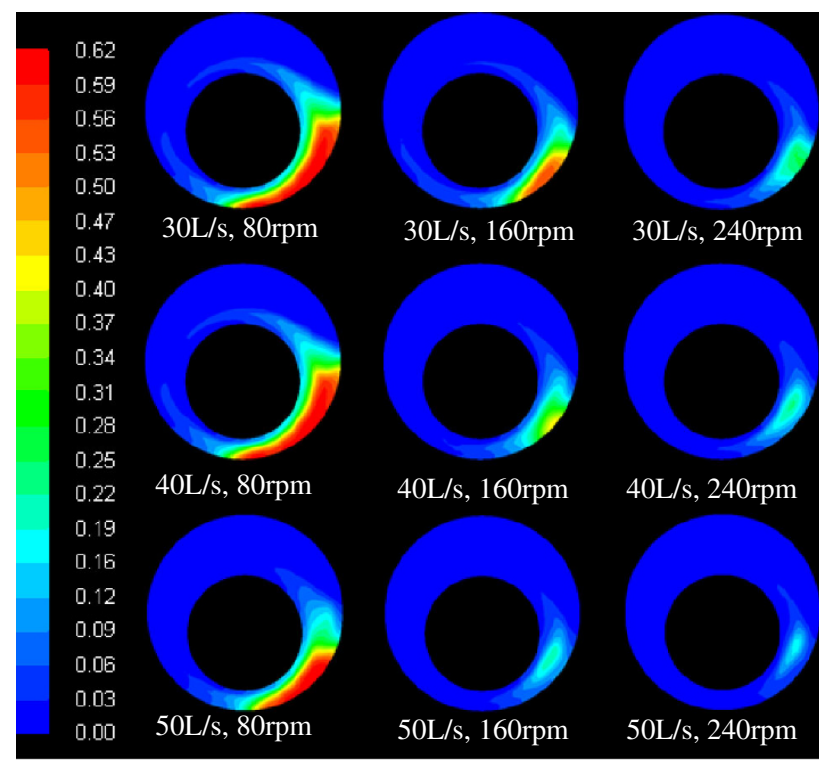

Fig. 5 Annular cuttings concentration contour at the angle of $60^{\circ}$

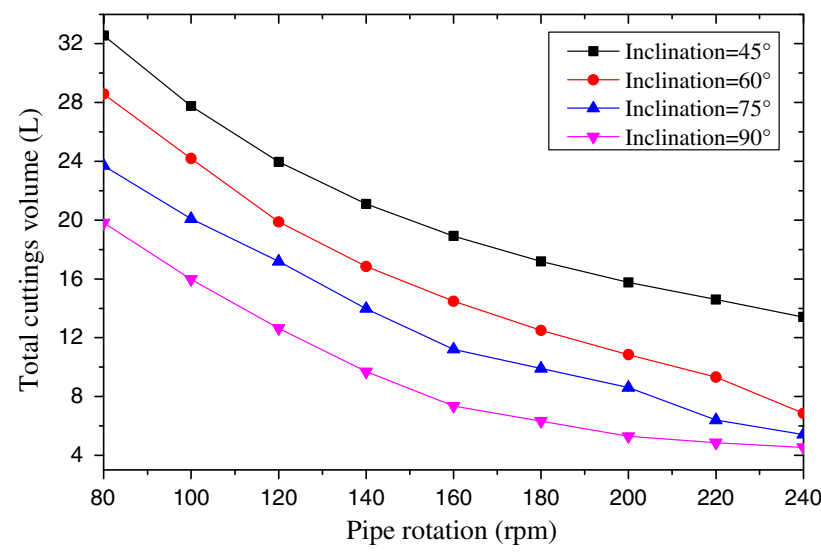

Fig. 6 Effect of pipe rotation on cuttings volume for different inclinations and flow rate $30 \mathrm{~L} / \mathrm{s}$

increases in the flow rate, all particles will be transported in a heterogeneous flow.

Effects of pipe rotation on cuttings concentration and pressure drop

As seen from Figs. 6, 7, 8, 9, at fluid rates from 30 to $50 \mathrm{~L} / \mathrm{s}$ and inclinations horizontal to $60^{\circ}$, pipe rotation can significantly decrease the total volume of annular cuttings. Even though this is the case, still a decrease in the cuttings volume is observed with the increase of rotation speed. However, the additional contribution of drillpipe rotation on decreasing cuttings volume is almost negligible after a certain rotation speed when flow rate reaches $50 \mathrm{~L} / \mathrm{s}$ or inclination is close to horizontal. Considering the limited rotation speed in real drilling, pipe rotation has a significant

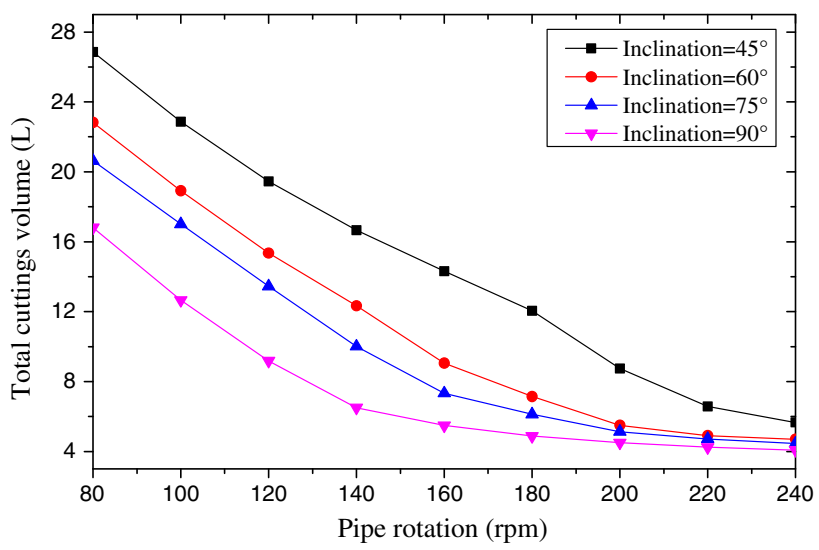

Fig. 7 Effect of pipe rotation on cuttings volume for different inclinations and flow rate $40 \mathrm{~L} / \mathrm{s}$

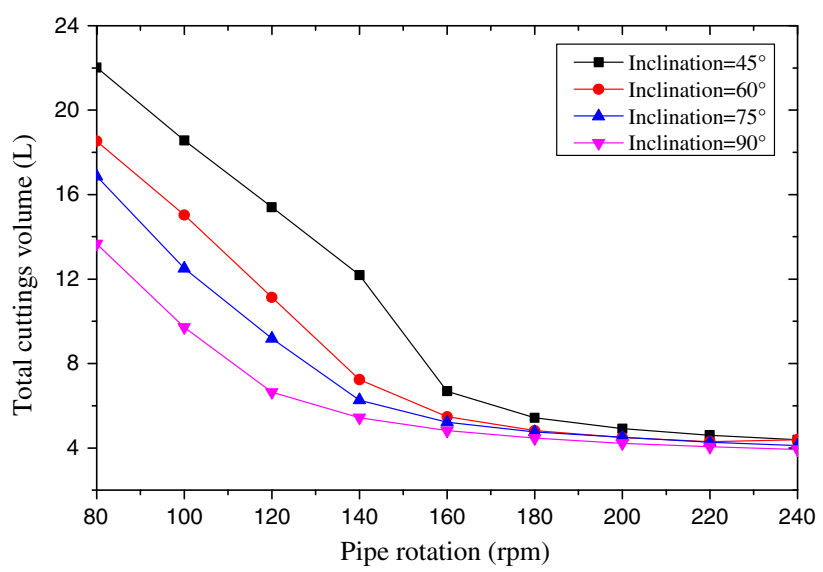

Fig. 8 Effect of pipe rotation on cuttings volume for different inclinations and flow rate $50 \mathrm{~L} / \mathrm{s}$

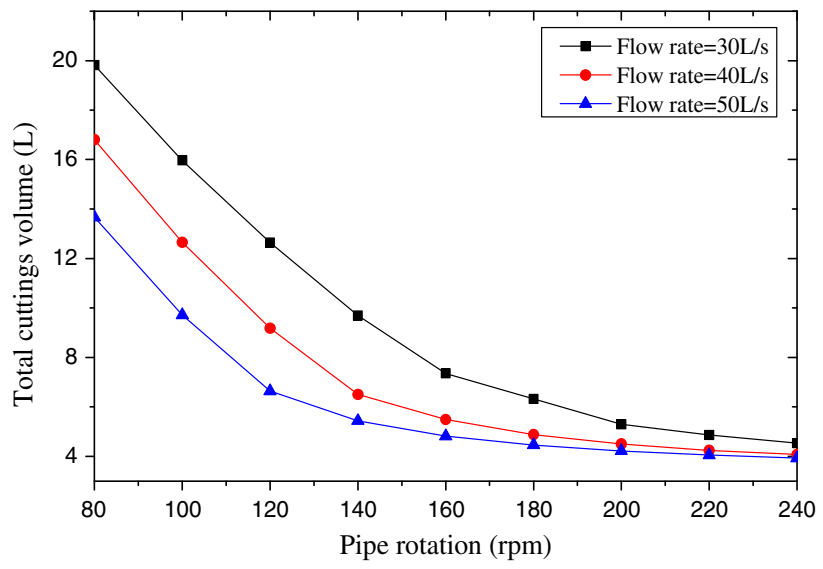

Fig. 9 Effect of pipe rotation on cuttings volume for different flow rates in horizontal wellbores

impact on hole cleaning in low flow rates. In addition, the pipe rotation effect on hole cleaning is also affected by inclination. In particular, in the lower pipe rotation speeds, the cuttings volume in the annulus is more for lower 


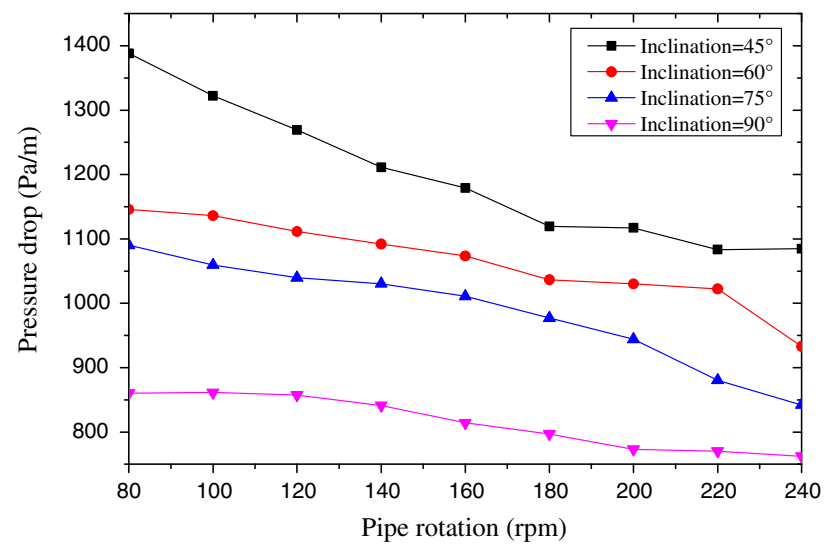

Fig. 10 Effect of pipe rotation on annular pressure drop for different inclinations and flow rate $30 \mathrm{~L} / \mathrm{s}$

inclinations than for higher inclinations, which means that cuttings bed is easier to be formed in the low inclination. This conclusion is consistent with Li et al.'s (2001) observation that the toughest section for hole cleaning is the build section rather than the vertical or the horizontal section.

As shown from Figs. 10, 11, 12, the influences of drillpipe rotation for different flow rates and inclinations on annular pressure drop are depicted. For any flow rate and inclination, as the pipe rotation speed is increased, annular pressure drop is decreased. The pressure drop almost reduces by half when pipe rotation increases from 80 to $160 \mathrm{rpm}$ with $50 \mathrm{~L} / \mathrm{s}$ flow rate at $45^{\circ}$. But when keeping flow rate $50 \mathrm{~L} / \mathrm{s}$, the pressure drop almost remains constant after pipe rotation speed reaches $180 \mathrm{rpm}$. Also, in the low rotation speed, producing pressure drop becomes more at the lower angle than at higher angle.

\section{Empirical correlations}

It is considered that the ratio of cuttings volume and wellbore volume is function of inclination, flow rate, density and size of cuttings, viscosity and density of drilling fluid, annular hydraulic diameter, pipe rotation, and gravity. Major parameters affecting cuttings concentration can be defined as:

$\frac{V_{\mathrm{b}}}{V_{\mathrm{W}}}=f\left(\theta, v, \omega, \rho_{l}, \rho_{s}, \mu, D_{\mathrm{h}}, g, d_{\mathrm{c}}\right)$

Based on Buckingham- $\pi$ theorem, seven dimensionless groups are determined as follows (Ozbayoglu et al. 2008):

$$
\begin{aligned}
& \pi_{1}=\frac{\rho_{l} v D_{\mathrm{h}}}{\mu} \\
& \pi_{2}=\frac{v^{2}}{g D_{\mathrm{h}}} \\
& \pi_{3}=C \\
& \pi_{4}=\theta
\end{aligned}
$$

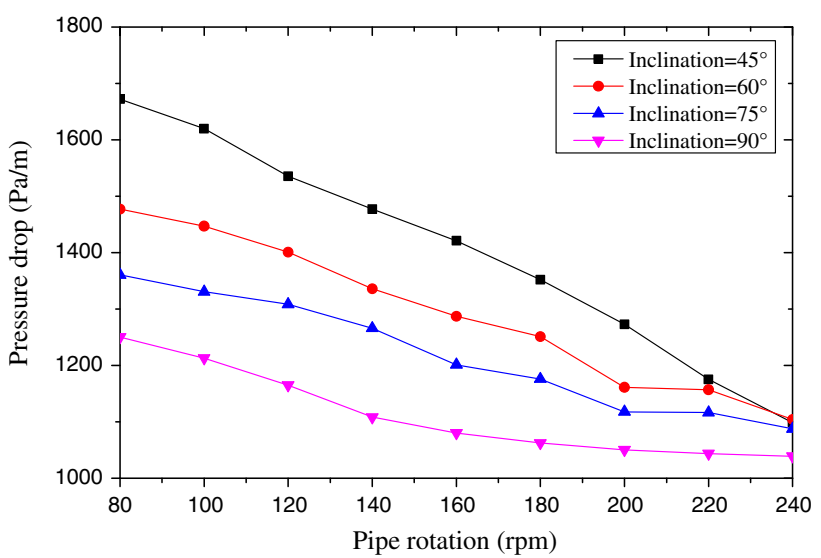

Fig. 11 Effect of pipe rotation on annular pressure drop for different inclinations and flow rate $40 \mathrm{~L} / \mathrm{s}$

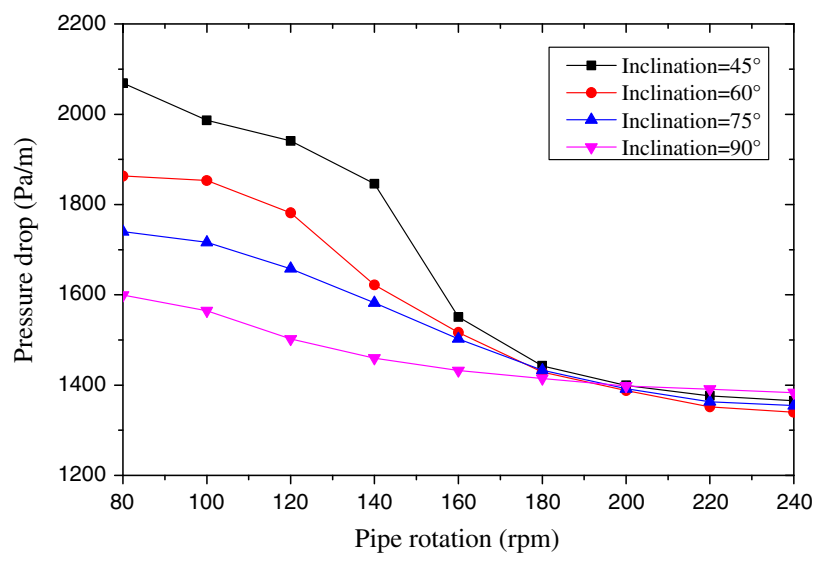

Fig. 12 Effect of pipe rotation on annular pressure drop for different inclinations and flow rate $50 \mathrm{~L} / \mathrm{s}$

$\pi_{5}=\frac{d_{\mathrm{c}}}{D_{\mathrm{h}}}$

$\pi_{6}=\frac{\rho_{l} v d_{\mathrm{c}}}{\mu}$

$\pi_{7}=\frac{\omega D_{\mathrm{h}}}{v}$

where $V_{\mathrm{b}}$ represents cuttings volume $\left(\mathrm{m}^{3}\right), V_{\mathrm{w}}$ is wellbore volume $\left(\mathrm{m}^{3}\right), \theta$ is the inclination $\left(^{\circ}\right), v$ represents fluid flow rate $\mathrm{m} / \mathrm{s}, \omega$ stands for pipe rotation $\left(\mathrm{s}^{-1}\right), \rho_{l}$ is the density of drilling fluid $\left(\mathrm{kg} / \mathrm{m}^{3}\right), \rho_{s}$ is the density of cuttings $\left(\mathrm{kg} / \mathrm{m}^{3}\right), \mu$ represents the viscosity of drilling fluid (Pa s), $d_{\mathrm{c}}$ is the cuttings size $(\mathrm{m}), D_{\mathrm{h}}$ stands for the hydraulic diameter (m), $C$ is the cuttings concentration, $g$ stands for the gravity $(\mathrm{N} / \mathrm{kg})$.

In this simulation, size and density of cuttings, injected cuttings concentration, viscosity and density of drilling fluid are constant, therefore, $\pi_{3}, \pi_{5}$, and $\pi_{6}$ can be negligible, and Eq. (5) is rewritten by: 
$\frac{V_{\mathrm{b}}}{V_{\mathrm{W}}}=a_{0} \pi_{1}^{a_{1}} \pi_{2}^{a_{2}} \pi_{4}^{a_{3}} \pi_{7}^{a_{4}}$

Considering the effect of cuttings concentration, annular pressure drop can be defined as:

$\frac{\Delta p}{\Delta L}=a_{0} \pi_{1}^{a_{1}} \pi_{2}^{a_{2}} \pi_{4}^{a_{3}} \pi_{7}^{a_{4}}\left(\frac{V_{\mathrm{b}}}{V_{\mathrm{W}}}\right)^{a_{5}}$

The least square method is applied to linearly regress for 12 groups data, and cuttings concentration and annular pressure drop for inclinations from $45^{\circ}$ to $90^{\circ}$ can be determined, respectively:

$$
\begin{aligned}
\frac{V_{\mathrm{b}}}{V_{\mathrm{W}}}= & 0.01\left(\frac{\rho_{l} v D_{\mathrm{h}}}{1,000 \mu}\right)^{6.0493}\left(\frac{v^{2}}{g D_{\mathrm{h}}}\right)^{-4.3133} \\
& \times(0.1 \theta)^{-0.8977}\left(\frac{10 \omega D_{\mathrm{h}}}{v}\right)^{-1.3677} \\
\frac{\Delta p}{\Delta L}= & 100\left(\frac{\rho_{l} v D_{\mathrm{h}}}{1,000 \mu}\right)^{1.0547}\left(\frac{v^{2}}{g D_{\mathrm{h}}}\right)^{0.1376}(0.1 \theta)^{-0.0740} \\
& \times\left(\frac{10 \omega D_{\mathrm{h}}}{v}\right)^{0.1435}\left(\frac{100 V_{\mathrm{b}}}{V_{\mathrm{W}}}\right)^{0.2877}
\end{aligned}
$$

\section{Conclusions}

In this study, CFD simulations of the effects of drillpipe rotation on cuttings transport behavior in the complex structure well were performed using an Euler multiphase model. The following conclusions can be drawn as follows:

- Pipe rotation has an important effect on distribution of annular cuttings in inclined section and causes the asymmetrical distribution of cuttings along the circumference direction.

- Pipe rotation can significantly increase the tangential velocity of drilling fluid, which generates the drag force along the tangential direction, and the force can restrain cuttings bed development to some extent. In high rotation speeds, dragged effect is more pronounced, and the fully suspended flow is easy to be developed.

- At low or medium flow rates, increasing pipe rotation speed can significantly reduce cuttings volume and annular pressure drop. However, there exists a threshold point at high flow rates, after which no matter how much the pipe rotation speed is increased, the magnitudes of cuttings volume and annular pressure drop almost remain constant.

- Buckingham- $\pi$ theorem was applied to define dimensionless groups, and eventually, user friendly empirical correlations about the cuttings concentration and pressure drop were developed using least square method based on the CFD simulations on cuttings transport.

Acknowledgments This work was supported by Program for National Science and Technology Major Project of China (No. 2011ZX05009-005) and the National Natural Science Foundation of China (Nos. 51174043, 51204056) are gratefully acknowledged.

Open Access This article is distributed under the terms of the Creative Commons Attribution License which permits any use, distribution, and reproduction in any medium, provided the original author(s) and the source are credited.

\section{References}

Bassal AA (1996) The effect of drillpipe rotation on cuttings transport in inclined wellbores. MS Thesis, University of Tulsa

Bilgesu HI, Ali MW et al. (2002) Computational fluid dynamics (CFD) as a tool to study cutting transport. In: SPE Eastern Regional Meeting, Lexington

Bilgesu HI, Mishra N et al. (2007) Understanding the effects of drilling parameters on hole cleaning in horizontal and deviated wellbores using computational fluid dynamics. In: SPE Eastern Regional Meeting, Lexington

Duan MQ, Miska S et al (2008) Transport of small cuttings in extended reach drilling. SPE Drill Complet 23(3):258-265

Li J, Walker S (2001) Sensitivity analysis of hole cleaning parameters in directional wells. SPE J 6(4):356-363

Li M, Jv YF et al (2010) Simulation on the effect of drillstring rotation on hole cleaning for extended reach well. West-China Explor Eng 5:51-54

Mishra N (2007) Investigation of hole cleaning parameters using computational fluid dynamics in horizontal and deviated wells. MS Thesis, West Virginia University

Ozbayoglu ME, Saasen A et al. (2008). Effect of pipe rotation on hole cleaning for water-based drilling fluids in horizontal and deviated wells. In: IADC/SPE Asia Pacific drilling technology conference and exhibition, SPE, Jakarta, Indonesia

Peden JM, Ford JT et al. (1990) Comprehensive experimental investigation of drilled cuttings transport in inclined wells including the effects of rotation and eccentricity. In: European Petroleum Conference, SPE, Hague, Netherlands

Sanchez RA, Azar JJ et al (1999) Effect of drillpipe rotation on hole cleaning during directional-well drilling. SPE J 4(2):101-108

Shih TH, Liou WW et al (1995) A new $\kappa-\varepsilon$ eddy viscosity model for high Reynolds number turbulent flows. Comput Fluids 24(3):227-238

Sifferman TR, Becker TR (1992) Hole cleaning in full-scale inclined wellbores. SPE Drill Eng 7(2):115-120

Sorgun M (2010) Modeling of Newtonian fluids and cuttings transport analysis in high inclination wellbores with pipe rotation. Ph. D Thesis, Middle East Technical University

Syamlal M, O'Brien TJ (1989) Computer simulation of bubbles in a fluidized bed. AIChE Symp Series 85:22-31

Wang ZM, Guo XL et al (2009) Effect of drillpipe rotation on borehole cleaning for extended reach well. J Hydrodyn 21(3):366-372 